\title{
Expression of miR-664 and miR-184 on proliferation, apoptosis and migration of osteosarcoma cells
}

\author{
PENGFEI TAO ${ }^{1 *}$, JING FENG $^{1 *}$, QIONG LI $^{2}$, WEI LIU ${ }^{1}$, LIN YANG $^{1}$, \\ XIAOLONG ZHAO ${ }^{1}$, HUAN NI $^{1}$ and PING XIA ${ }^{1}$ \\ ${ }^{1}$ Department of Spine Surgery, Wuhan No. 1 Hospital, Wuhan, Hubei 430022; \\ ${ }^{2}$ Department of Radiology, Taihe Hospital Hubei University of Medicine, Shiyan, Hubei 442000, P.R. China
}

Received April 25, 2018; Accepted November 1, 2018

DOI: $10.3892 / 01.2018 .9739$

\begin{abstract}
The expression of micro-ribonucleic acid miR-664 and miR-184 on the biological characteristics of osteosarcoma (OS) SOSP-9607 cells was investigated. Eighteen surgical specimens of OS and 18 normal tissue specimens were collected. The expression of miR- 664 and miR-184 was detected via fluorescence reverse transcription-quantitative polymerase chain reaction (RT-qPCR). The OS cell line SOSP-9607 was selected as the object of study, and miR-664 blank group, miR-664 mimic group, miR-664 inhibitor group, miR-184 blank group, miR-184 mimic group and miR-184 inhibitor group were established through transfection. Changes in apoptosis were detected via flow cytometry, the cell proliferation capacity was detected via Cell Counting Kit- 8 assay, and the cell migration was observed via wound healing assay. In cancer tissues of OS patients, the relative expression of miR-664 and miR-184 was significantly higher than that in para-carcinoma tissues $(\mathrm{P}<0.05)$. The cell growth in miR-664 inhibitor group was obviously decreased compared with those in miR-664 blank and mimic groups $(\mathrm{P}<0.05)$. There were differences in the cell migration capacity among groups $(\mathrm{P}<0.01)$, and the cell scratch areas in miR-664 and miR-184 mimic groups were significantly decreased compared with those in miR-664 and miR-184 blank groups $(\mathrm{P}<0.05)$, while they were significantly increased in miR-664 and miR-184 inhibitor groups compared with those in miR-664 and miR-184 blank and mimic groups $(\mathrm{P}<0.05, \mathrm{P}<0.01)$. There were differences in the apoptosis rate among groups $(\mathrm{P}<0.01)$ and apoptosis in miR-664 and miR-184 inhibitor groups was remarkably increased compared with those in miR-664 and miR-184 blank and mimic groups
\end{abstract}

Correspondence to: Dr Ping Xia, Department of Spine Surgery, Wuhan No. 1 Hospital, 215 Zhongshan Road, Wuhan, Hubei 430022, P.R. China

E-mail:pvr9uj@163.com

${ }^{*}$ Contributed equally

Key words: miR-664, miR-184, osteosarcoma, SOSP-9607, migration, apoptosis, proliferation
$(\mathrm{P}<0.05)$. Downregulating the expression of miR-664 and miR-184 may promote apoptosis, inhibit the proliferation and reduce the migration capacity of SOSP-9607 cells. Therefore, miR-664 and miR-184 may provide a theoretical basis for the target selection in clinical targeted therapy and drug development for OS.

\section{Introduction}

Osteosarcoma (OS) is a malignant tumor frequently occurring in teenagers, which mainly occurs at the site of bone turnover and rapid growth, and the most typical site is metaphysis of long bone in teenage patients (1). According to studies (2), the incidence of OS is divided into two high-frequency stages: primary OS is dominated in teenagers aged $\sim 12$ years, while secondary OS is dominated in the elderly aged $\sim 75$ years. More than $80 \%$ OS will metastasize, and local invasion and metastasis have already occurred possibly when the disease is found in the early stage. The metastatic site of OS is mainly the lungs, and it may metastasize to the kidney, brain and prostate, in a small number of patients $(3,4)$. At present, surgical resection dominates in the recognized therapeutic regimens for OS, and patients need to receive chemotherapy before and after surgery. However, other secondary malignant tumors occur easily in patients during chemotherapy, and OS is likely to relapse in some patients, which is also the main reason for the 5-year survival rate of only $15-20 \%$ in OS patients (5). Therefore, optimizing the treatment and diagnosis of OS patients and improving the long-term survival rate of OS patients are problems demanding a prompt solution.

With the social progress and rapid development of science and technology, there has been increasing research on cancer from the perspective of molecular biology. Micro-ribonucleic acid (miRNA) is a single-stranded deoxyribonucleic acid (DNA) molecule encoded by the endogenous gene, which is as short as $22 \mathrm{nt}$ and mainly exists in plants, animals and DNA virus (6). Moreover, miRNA is an important non-protein-coding RNA, which regulates the target messenger RNA (mRNA) of gene expression and triggers or inhibits translation or mRNA cleavage (7). Studies have revealed (8) that miR-184, as one of the important members of miRNAs, is closely related to the occurrence and development of a variety of tumors. However, miR-664 is less reported, and, according to literature (9), it has 
certain correlations with diabetes mellitus, acute lymphatic leukemia and melanoma. Whether there is a differential expression of miR-184 and miR-664 in OS and whether it has effects on the biological functions of OS remains unclear.

Therefore, in this study, the expression of miR-184 and miR-664 in cancer tissues of OS patients was detected, and miR-184 and miR-664 mimics and inhibitors were transfected into OS cell line SOSP-9607, so as to observe their effects on the biological behavior of OS and provide a new theory and basis for clinical treatment.

\section{Patients and materials}

A total of 18 OS patients treated in Wuhan No. 1 Hospital (Wuhan, China) from July 2013 to January 2015 were collected, including 11 males and 7 females aged 13-22 years with an average age of $15.34 \pm 2.51$ years. During surgery, cancer and para-carcinoma tissues were collected from patients, and stored at $-80^{\circ} \mathrm{C}$ within $5-10$ min for standby application. This study was approved by the Medical Ethics Committee of Wuhan No. 1 Hospital, and patients or their families were informed and signed the informed consent. Patients in this study all met the diagnostic criteria for OS (10). SOSP-9607 cells used in this study were purchased from the Laboratory of Orthopedics, Tangdu Hospital, the Fourth Military Medical University (Xi'an, China).

Inclusion and exclusion criteria. Inclusion criteria: patients with a course of disease of more than half a year; patients without recent anticancer drug therapy; patients without other genetic diseases; patients without receiving radiotherapy and chemotherapy and patients with complete clinical data.

Exclusion criteria: patients with respiratory diseases, hypertension or diabetes mellitus; patients with blood relation; patients without receiving blood transfusion therapy recently or patients with autism, memory disorder, hearing disorder and limb impairment and patients who did not cooperate in follow-up and whose clinical data were incomplete.

Main reagents and instruments. Roswell Park Memorial Institute (RPMI)-1640 medium, fetal bovine serum (FBS), trypsin, and penicillin-streptomycin double antibiotics were purchased from Gibco (Thermo Fisher Scientific, Inc., Waltham, MA, USA). The Cell Counting Kit-8 (CCK-8) was purchased from Shanghai Beyotime Institute of Biotechnology (Shanghai, China). TRIzol reagent, PrimeScript RT kit and Lipofectamine $^{\mathrm{TM}} 2000$ transfection kit were purchased from Invitrogen (Thermo Fisher Scientific, Inc.). SYBR Premix Ex Taq II was purchased from Takara Bio Inc. (Otsu, Japan). The Annexin V-fluorescein isothiocyanate (FITC) apoptosis assay kit was purchased from BD Biosciences (San Jose, CA, USA). Flow cytometer was purchased from BD Biosciences, and reverse transcription-quantitative polymerase chain reaction (RT-qPCR) instrument was purchased from ABI (Thermo Fisher Scientific, Inc.). miR-664 and miR-184 primer sequences and inhibitors were provided by Sangon Biotech Co., Ltd. (Shanghai, China).

Cell culture. SOSP-9607 cells were cultured in the RPMI-1640 medium containing $100 \mathrm{U} / \mathrm{ml}$ penicillin and $100 \mu \mathrm{g} / \mathrm{ml}$ streptomycin double antibodies and 10\% FBS in a thermostatic incubator with $5 \% \mathrm{CO}_{2}$ at $37^{\circ} \mathrm{C}$. When SOSP-9607 cells grew to the logarithmic growth phase, the cell density was adjusted to $5 \times 10^{5} /$ well followed by taking out cells, cells were inoculated into a 6-well plate, and the cell fusion was observed. When $70-80 \%$ cells were fused, SOSP-9607 cells were transfected using the Lipofectamine ${ }^{\mathrm{TM}} 2000$ transfection kit, and divided into miR-664 blank group (transfected with empty plasmid), miR-664 mimic group (transfected with miR-664 mimic), miR-664 inhibitor group (transfected with miR-664 inhibitor), miR-184 blank group (transfected with empty plasmid), miR-184 mimic group (transfected with miR-184 mimic), and miR-184 inhibitor group (transfected with miR-184 inhibitor). After transfection for $48 \mathrm{~h}$, cells were collected for subsequent experiments.

Cell proliferation. Cell proliferation was detected via CCK-8 assay. After transfection for $48 \mathrm{~h}$, cells in the 6 groups were inoculated into a 96 -well plate at a density of $2 \times 10^{3} /$ well, and cell proliferation was detected at $0,24,48,72$ and $96 \mathrm{~h}$ after culture, respectively. A total of $10 \mu \mathrm{l} \mathrm{CCK}-8$ solution was added into each well for incubation at $37^{\circ} \mathrm{C}$ for $2 \mathrm{~h}$, and the absorbance was measured at a wavelength of $450 \mathrm{~nm}$ using a microplate reader (Bio-Rad Laboratories, Inc., Hercules, CA, USA). Three repeated wells were set in each group, the experiment was repeated 3 times, and the average was taken in experimental results.

Cell migration. After transfection, cells in the 6 groups were inoculated into a $24-$ well plate at a density of $1 \times 10^{5} /$ well. The bottom of the culture plate was scratched using a sterile pipette $(200 \mu \mathrm{l})$. The migration of cell scratch was observed at $36 \mathrm{~h}$ using an inverted microscope (Olympus, Tokyo, Japan), and the cell migration capability was compared. Three repeated wells were set in each group, the experiment was repeated 3 times, and the average was taken in experimental results.

Apoptosis assay. After transfection, the culture solution in the 6 groups of cells was discarded, and cells were washed with phosphate-buffered saline (PBS) and digested with $0.25 \%$ trypsin, followed by centrifugation at $780 \mathrm{xg}$ for $5 \mathrm{~min}$ using a low-speed centrifuge. Then the supernatant was discarded, and cells were collected, washed with the incubation buffer, resuspended and added with Annexin V-FITC, followed by incubation in the dark at room temperature for $15 \mathrm{~min}$. Cells were washed again with the buffer and apoptosis was detected using a flow cytometer. Three repeated wells were set in each group, the experiment was repeated 3 times, and the average was taken in experimental results.

$R T$ - $q P C R$. RNA was extracted from cells and tissues according to the instructions of the TRIzol reagent kit, and the mass and concentration of RNA extracted were detected using a spectrophotometer (Bio-Rad Laboratories, Inc.). RNA was reverse transcribed into complementary DNA (cDNA) using the PrimeScript RT kit, after which part of cDNA was taken for subsequent experiments. Then amplification was performed using ABI Prism 7900, and the amplification system is as follows: $12.5 \mu 1$ SYBR Premix Ex Taq II (2X), $1.0 \mu 1$ forward primer, $1.0 \mu \mathrm{l}$ reverse primer, $2.0 \mu \mathrm{l} \mathrm{cDNA}$, addition of double 
Table I. Primer sequences.

\begin{tabular}{lll}
\hline Genes & \multicolumn{1}{c}{ Forward primers } & \multicolumn{1}{c}{ Reverse primers } \\
\hline$m i R-664$ & 5'-TACAACACCGGTCACTAACGCATTG-3' & 5'-GTATCACCTCCTCCAGCAACTAACA-3' \\
$m i R-184$ & 5'-TACGACTATGTGACCTGCCTG-3' & 5'-TGGTTCAACTCTCCTTTCCA-3' \\
GAPDH & 5'-GTCTCCTCTGACTTCAACAGCG-3' & 5'-ACCACCCTGTTGCTGTAGCCAA-3'
\end{tabular}

GAPDH, glyceraldehyde-3-phosphate dehydrogenase.
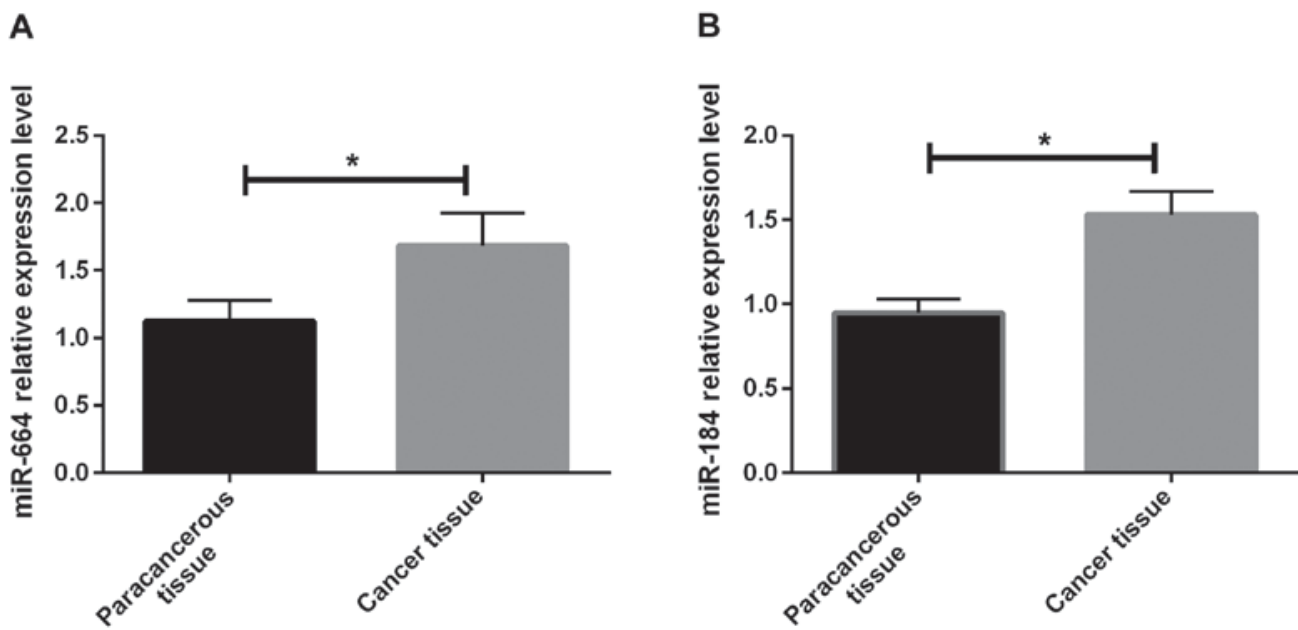

Figure 1. (A) Expression of miR-664 in tissues of OS patients. RT-qPCR shows that the expression of miR-664 in cancer tissues of patients is significantly higher than that in para-carcinoma tissues, and there is a statistically significant difference $(\mathrm{t}=3.346, \mathrm{P}=0.029)$. ${ }^{*} \mathrm{P}<0.05$, there is a difference between the two groups. (B) Expression of miR-184 in tissues of OS patients. RT-qPCR displays that the expression of miR-184 in cancer tissues of patients is significantly higher than that in para-carcinoma tissues, and there is a statistically significant difference $(t=6.362, P=0.003)$. ${ }^{*} \mathrm{P}<0.05$, there is a difference between the two groups. OS, osteosarcoma.

distilled water to total volume of $25 \mu \mathrm{l}$. Amplification conditions are as follows: pre-denaturation at $95^{\circ} \mathrm{C}$ for $2 \mathrm{~min}, 95^{\circ} \mathrm{C}$ for $30 \mathrm{sec}, 60^{\circ} \mathrm{C}$ for $35 \mathrm{sec}, 72^{\circ} \mathrm{C}$ for $1 \mathrm{~min}$, a total of 40 cycles. Glyceraldehyde-3-phosphate dehydrogenase (GAPDH) was used as an internal reference, and results are presented as $2^{-\triangle \mathrm{Cq}}(11)$. Three repeated wells were set in each group, the experiment was repeated 3 times, and the average was taken in experimental results (Table I).

Statistical analysis. In this study, GraphPad Prism 7 software (GraphPad Software, Inc., La Jolla, CA, USA) was used for drawing, and Statistical Product and Service Solutions (SPSS) 20.0 software (SPSS Inc., Chicago, IL, USA) was used for statistical analysis of collected data. Measurement data were presented as mean \pm standard deviation (mean \pm SD). t-test was used for the comparison of means between two groups, and one-way analysis of variance was used for the comparison among groups and the post hoc test was Least Significant Difference test. $\mathrm{P}<0.05$ was considered to indicate a statistically significant difference.

\section{Results}

Expression of $m i R-664$ and $m i R-184$. In this study, the relative expression of miR-664 and miR-184 in cancer and para-carcinoma tissues of patients was detected via RT-qPCR.
Results showed that the relative expression of miR-664 and miR-184 in cancer tissues of patients was significantly higher than that in para-carcinoma tissues, and there were statistically significant differences $(\mathrm{P}<0.05)$ (Fig. 1A and $\mathrm{B})$. It was found in the detection of the relative expression of miR-664 and miR-184 in each group of cells after transfection that the relative expression of miR-664 and miR-184 in miR-664 and miR-184 inhibitor groups was obviously lower than that in the miR-664 and miR-184 blank and mimic groups, displaying statistically significant differences $(\mathrm{P}<0.05)$. Besides, the relative expression in the miR-664 and miR-184 blank groups was lower than that in the miR-664 and miR-184 mimic groups $(\mathrm{P}<0.05)$ (Fig. 2A and B).

Cell proliferation after transfection. The cell proliferation capacity in each group was detected via CCK-8 assay after transfection. Results manifested that there were no differences in the cell growth among miR-664 groups within 24-72 h after cell transfection $(\mathrm{P}>0.05)$, there was no difference in the cell growth between the miR-664 mimic and blank groups at $96 \mathrm{~h}(\mathrm{P}>0.05)$, and the cell growth in the miR-664 inhibitor group was obviously decreased compared with that in the miR-664 blank and mimic groups, displaying significant differences $(\mathrm{P}<0.05)$ (Fig. 3A). Moreover, the observation of the miR-184 groups showed that there were no differences in the cell growth among miR-184 groups within 24-72 $\mathrm{h}$ after cell transfection $(\mathrm{P}>0.05)$, there was 
A

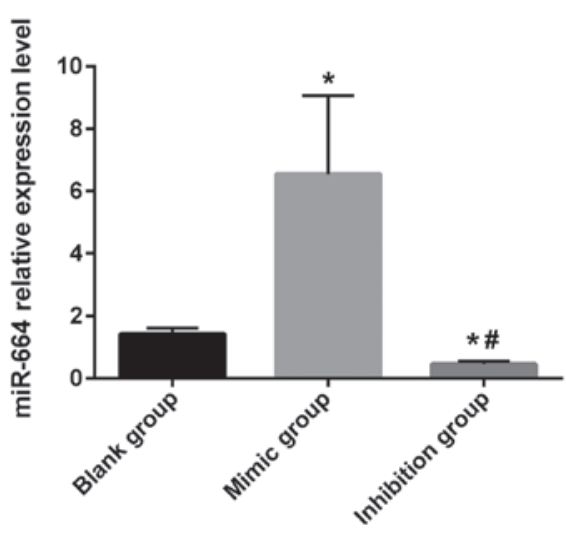

B

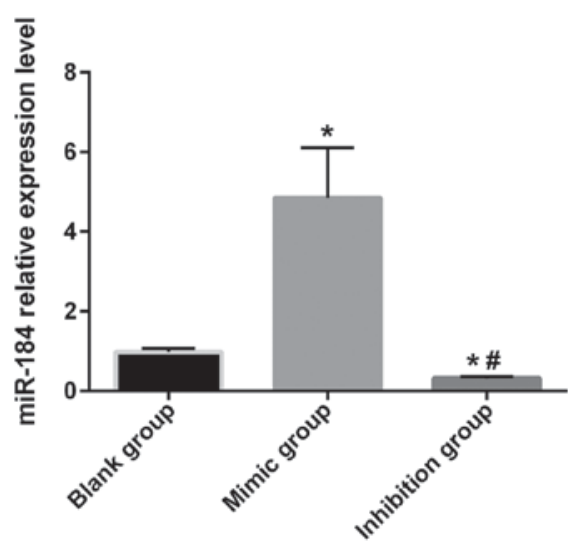

Figure 2. (A) The expression of miR-664 in each group after transfection. The relative expression of miR-664 in each group after transfection is detected via RT-qPCR. Results manifest that there are differences in the relative expression level of miR-664 after transfection among the groups ( $F=15.146, \mathrm{P}=0.005)$; the relative expression level of miR-664 in miR-664 inhibitor group is significantly lower than those in the blank and mimic groups, with statistically significant differences (inhibitor group vs. mimic group: $\mathrm{t}=8.106, \mathrm{P}=0.001$. Inhibitor group vs. blank group: $\mathrm{t}=4.192, \mathrm{P}=0.014$ ), and it is lower in the miR-664 blank group than that in the miR-664 mimic group $(\mathrm{t}=3.519, \mathrm{P}=0.025) .{ }^{*} \mathrm{P}<0.05$, there is a difference compared with the blank group. ${ }^{*} \mathrm{P}<0.05$, there is a difference compared with the mimic group. (B) The expression of miR-184 in each group after transfection. The relative expression of miR-184 in each group after transfection is detected via RT-qPCR. Results display that there are differences in the relative expression level of miR-184 after transfection among groups ( $\mathrm{F}=33.926$, $\mathrm{P}=0.001$ ), the relative expression of miR-184 in the miR-184 inhibitor group is obviously lower than that in the blank and mimic groups, with statistically significant differences (inhibitor group vs. mimic group: $\mathrm{t}=10.724, \mathrm{P}=0.001$. Inhibitor group vs. blank group: $\mathrm{t}=6.243, \mathrm{P}=0.003$ ), and it is lower in the miR-184 blank group than that in the miR-184 mimic group $(\mathrm{t}=5.322, \mathrm{P}=0.006) .{ }^{*} \mathrm{P}<0.05$, there is a difference compared with the blank group. ${ }^{*} \mathrm{P}<0.05$, there is a difference compared with the mimic group.

A

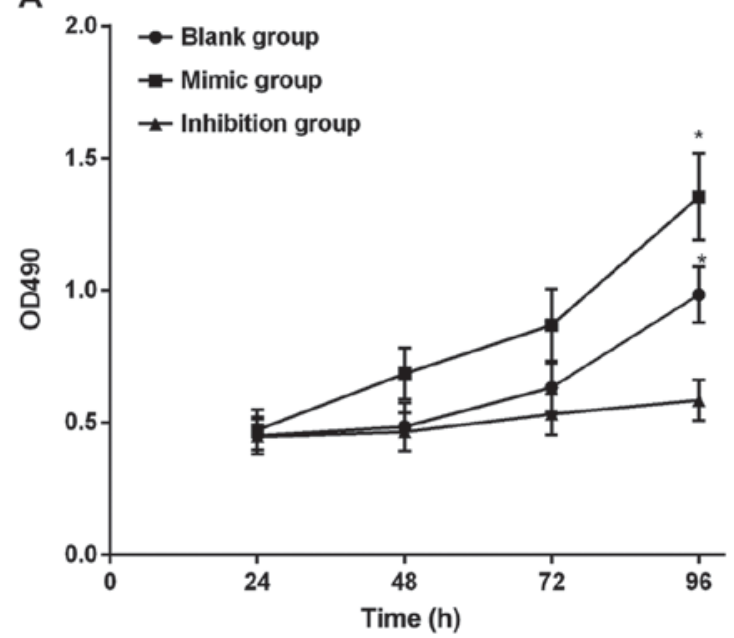

B

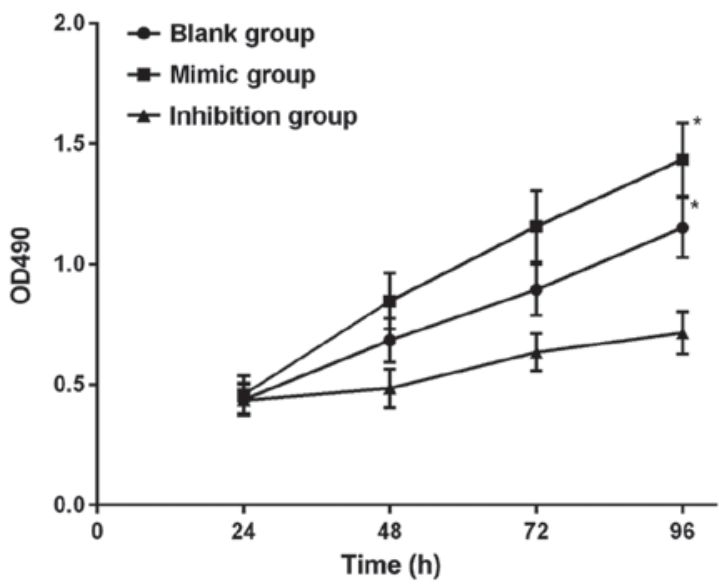

Figure 3. (A) Cell proliferation capacities in the miR-664 groups after transfection. According to the detection of cell proliferation capacity via CCK-8 assay, there are no differences in the cell growth among the miR-664 groups within 24-72 $\mathrm{h}$ after cell transfection ( $\mathrm{P}>0.05)$, but there are differences in the cell proliferation capacity among the miR-664 groups at $96 \mathrm{~h}(\mathrm{~F}=8.515, \mathrm{P}=0.018)$. The cell growth shows no difference between the miR-664 mimic and blank groups $(\mathrm{t}=1.445, \mathrm{P}=0.222)$, and it is obviously reduced in the miR-664 inhibitor group compared with that in the blank and mimic groups, with significant differences (inhibitor group vs. mimic group: $\mathrm{t}=4.094, \mathrm{P}=0.015$. Inhibitor group vs. blank group: $\mathrm{t}=2.868, \mathrm{P}=0.046$ ). ${ }^{*} \mathrm{P}<0.05$, there is a difference compared with the mimic group. (B) Cell proliferation capacities in the miR-184 groups after transfection. The detection of cell proliferation capacity via CCK-8 assay shows that there are no differences in the cell growth among the miR-184 groups within $24-72 \mathrm{~h}$ after cell transfection $(\mathrm{P}>0.05)$, but there are differences in the cell proliferation capacity among miR-184 groups at $96 \mathrm{~h}(\mathrm{~F}=10.076, \mathrm{P}=0.012)$. The cell growth shows no difference between the miR-184 mimic and blank groups $(\mathrm{t}=1.894$, $\mathrm{P}=0.131$ ), and it is obviously reduced in the miR-184 inhibitor group compared with that in the blank and mimic groups, with significant differences (inhibitor group vs. mimic group: $\mathrm{t}=4.247, \mathrm{P}=0.013$. Inhibitor group vs. blank group: $\mathrm{t}=3.044, \mathrm{P}=0.038)$. * $\mathrm{P}<0.05$, there is a difference compared with the mimic group.

no difference in the cell growth between the miR-184 mimic and blank groups at $96 \mathrm{~h}(\mathrm{P}>0.05)$, and the cell growth in the miR-184 inhibitor group was obviously decreased compared with that in the miR-184 blank and mimic groups, displaying significant differences $(\mathrm{P}<0.05)$ (Fig. 3A and $\mathrm{B})$.

Wound healing assay. Results of wound healing assay manifested that there were differences in the cell migration capacity among miR-664 groups $(\mathrm{P}<0.01)$, the cell scratch area in the miR-664 mimic group was significantly decreased compared with that in the miR-664 blank group, and there was a statistical difference between the two groups $(\mathrm{P}<0.05)$. The cell scratch area was significantly increased in the miR-664 inhibitor group compared with that in the miR-664 blank and mimic groups $(\mathrm{P}<0.05, \mathrm{P}<0.01)$. Moreover, the observation of the miR-184 groups demonstrated that there were differences 
Table II. Cell migration capacity.

\begin{tabular}{|c|c|c|c|c|c|c|c|}
\hline Group & Scratch area & F-value & P-value & Group & Scratch area & F-value & $\mathrm{P}$-value \\
\hline miR-664 blank & $45.51 \pm 4.58 \% \%^{\mathrm{a}, \mathrm{b}}$ & 83.759 & 0.001 & miR-184 blank & $55.84 \pm 6.54 \%{ }^{\mathrm{a}, \mathrm{b}}$ & 53.003 & 0.001 \\
\hline miR-664 inhibitor & $84.62 \pm 8.32 \% \mathrm{a}^{\mathrm{a}}$ & & & miR-184 inhibitor & $77.58 \pm 9.33 \% \%^{\mathrm{a}}$ & & \\
\hline miR-664 mimic & $23.58 \pm 3.54 \%$ & & & miR-184 mimic & $18.65 \pm 4.58 \%$ & & \\
\hline
\end{tabular}

${ }^{\mathrm{a}} \mathrm{P}<0.05$, compared with the mimic group. ${ }^{\mathrm{b}} \mathrm{P}<0.05$, compared with inhibitor group.

A

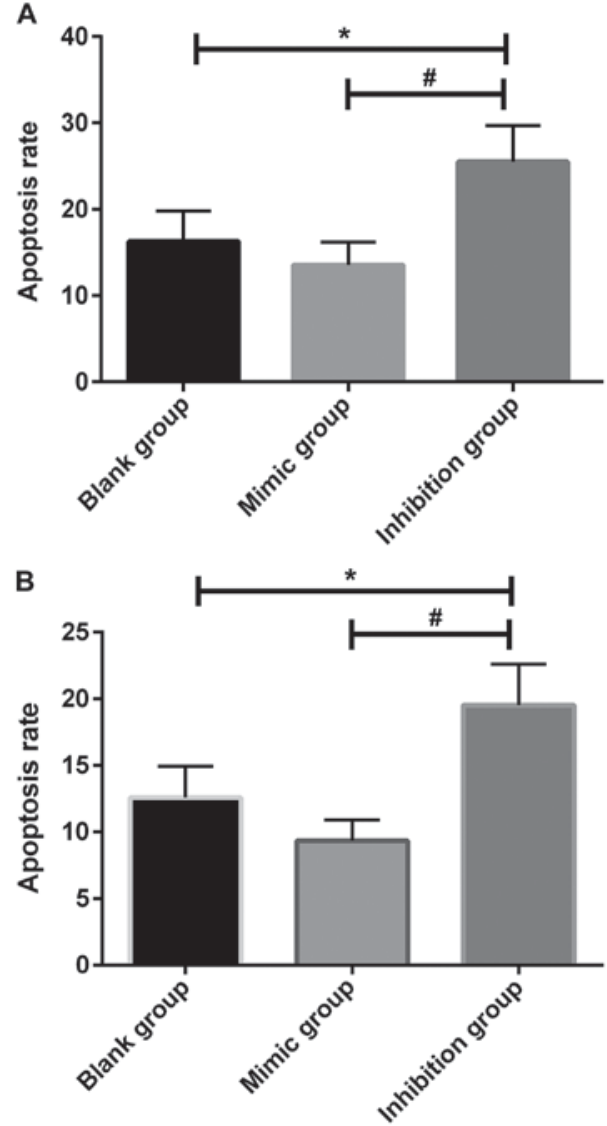

Figure 4. (A) Apoptosis in the miR-664 groups after transfection. According to the detection using the flow cytometer, there are differences in the apoptosis rate among the miR-664 groups after transfection $(\mathrm{F}=14.167, \mathrm{P}=0.005)$. Apoptosis has no significant difference in the miR-664 mimic group compared with that in the miR-664 blank group ( $\mathrm{t}=1.987, \mathrm{P}=0.118)$; it is obviously increased in the miR-664 inhibitor group compared with that in the miR-664 blank group ( $\mathrm{t}=3.131, \mathrm{P}=0.035)$, and it is decreased in the miR-664 mimic group compared with that in the miR-664 inhibitor group, displaying a significant difference $(t=5.159, \mathrm{P}=0.006) .{ }^{*} \mathrm{P}<0.01$, there is a difference compared with the blank group. ${ }^{~} \mathrm{P}<0.01$, there is a difference compared with the mimic group. (B) Apoptosis in the miR-184 groups after transfection. According to the detection using the flow cytometer, there are differences in the apoptosis rate among the miR-184 groups after transfection $(\mathrm{F}=9.431$, $\mathrm{P}=0.014)$. Apoptosis is significantly lower in the miR-184 mimic group than that in the miR-184 blank group ( $\mathrm{t}=1.072, \mathrm{P}=0.344)$; it is obviously increased in the miR-184 inhibitor group compared with that in the miR-184 blank group ( $\mathrm{t}=2.879, \mathrm{P}=0.044)$, and it is decreased in the miR-184 mimic group compared with that in the miR-184 inhibitor group, displaying a significant difference ( $\mathrm{t}=4.153, \mathrm{P}=0.014)$. ${ }^{*} \mathrm{P}<0.01$, there is a difference compared with the blank group. ${ }^{\prime \prime} \mathrm{P}<0.01$, there is a difference compared with the mimic group.

in the cell migration capacity among miR-184 groups $(\mathrm{P}<0.01)$. The cell scratch area in the miR-184 mimic group was remarkably smaller than that in the miR-184 blank group, and there was a statistical difference between the two groups $(\mathrm{P}<0.05)$. The cell scratch area was larger in the miR-184 inhibitor group than that in the miR-184 blank and mimic groups $(\mathrm{P}<0.05, \mathrm{P}<0.01)$ (Table II).

Apoptosis analysis. Apoptosis in each group of cells was detected using a flow cytometer. According to results, there were differences in the apoptosis rate among miR-664 groups $(\mathrm{P}<0.01)$, no statistically significant difference was found in apoptosis in the miR-664 mimic group compared with that in the miR-664 blank group ( $\mathrm{P}>0.05)$. Apoptosis in the miR-664 inhibitor group was remarkably increased compared with that in the miR-664 blank group $(\mathrm{P}<0.05)$, and it was reduced in miR-664 mimic group compared with that in the miR-664 inhibitor group, with a significant difference $(\mathrm{P}<0.01)$. Moreover, the comparisons among miR-184 groups demonstrated that no statistically significant difference was observed in apoptosis in the miR-184 mimic group compared with the miR-184 blank group ( $\mathrm{P}>0.05)$. Apoptosis in the miR-184 mimic group was remarkably lower than that in the miR-184 blank group $(\mathrm{P}<0.05)$, and it was obviously increased in the miR-184 inhibitor group compared with that in the miR-184 blank group $(\mathrm{P}<0.05)$, while it was reduced in the miR-184 mimic group compared with that in the miR-184 inhibitor group, with a significant difference $(\mathrm{P}<0.01)$ (Fig. 4A and $\mathrm{B})$.

\section{Discussion}

As the most common primary malignant bone tumor in clinic, OS possesses extremely strong invasion capacity, which has a certain metastasis trend in the early stage of disease, and lung metastasis occurs most easily in the early stage (12). Despite the continuous development and improvement of medical level, the comprehensive therapeutic method of limb salvage combined with chemotherapy increases the long-term survival rate of OS patients, but the survival rate always remains at approximately $60 \%$ without breakthroughs. Besides, the recurrence rate after surgery is more than $30 \%$, the treatment of patients with recurrence is very difficult, and the 5-year survival rate after treatment is only $20-30 \%(13,14)$. At present, the specific pathogenesis of OS remains unclear, and some studies have demonstrated that (15) there are many incidence reasons mainly dominated by genetic changes and environmental factors. Therefore, clarifying the related molecules to OS metastasis is of great significance in OS prevention and treatment, which is also a problem demanding prompt solution. 
As a small non-coding RNA, miRNA can regulate $30 \%$ of genes in the human body, and are also involved in a variety of cell physiological processes and occurrence and development of tumors, which plays an important role in vital activities of humans (16). In recent years, there have been many studies showing that the expression of miRNA is closely correlated with the clinical features and prognosis of tumors, including lung cancer (17), gastric cancer (18) and breast cancer (19). In addition, some other studies have proved that several kinds of miRNA influence the biological functions of OS (20). For example, Park et al reported that the low expression of miR-205 can serve as a prognostic index of early non-small cell lung cancer (21). Simonian et al found that the overexpression of miR-21 is related to the prognosis, lymph node metastasis and tumor node metastasis in gastric cancer patients in stages (22).

According to studies in recent years (23), the high expression of miR-664, a miRNA that is less reported and studied, in liver cancer can inhibit the expression of methionine adenosyltransferase $1 \mathrm{~A}$ and promote tumor proliferation and metastasis, whereas inhibiting miR-664 can induce the methionine adenosine transferase $1 \mathrm{~A}$ again and inhibit tumor growth and invasion, indicating that miR-664 is closely related to the tumor proliferation and migration. The expression of miR-184, a novel miRNA, in tumors is not clear yet, and its differential expression in different tumors has been proven in studies regulating the occurrence and development of tumors. For example, the high expression of miR-184 in glioma can promote the proliferation and invasion of glioma (24). However, whether the expression of miR-664 and miR-184 in OS play regulatory roles in the occurrence and development of OS remains to be clarified.

In this study, the relative expression levels of miR-664 and miR-184 in cancer and para-carcinoma tissues of OS patients were compared, and it was found that they were higher in cancer tissues than para-carcinoma tissues, suggesting that there are differences in the expression of miR-664 and miR-184 between cancer and para-carcinoma tissues of OS patients. According to the studies of Bao et al (23) and Lin et al (25), both miR-664 and miR-184 are highly expressed in OS, which is consistent with the results in this study. It is speculated that the high expression of miR-664 and miR-184 will change the biological functions of cells. However, little is known on how their expression affect the proliferation, migration and apoptosis of tumor cells. Therefore, in vitro experiment was performed. SOSP-9607 cells were transfected with miR-664 and miR-184 to successfully set up blank, mimic and inhibitor groups. CCK-8 assay, wound healing assay and flow cytometry demonstrated that the cell proliferation capacities in miR-664 and miR-184 blank groups were significantly lower than those in miR-664 and miR-184 mimic groups, and they were also significantly lower in miR-664 and miR-184 inhibitor groups than those in blank and mimic groups. The migration assay displayed that the cell migration capacities in miR-664 and miR-184 inhibitor groups were obviously lower than those in blank and mimic groups, indicating that inhibiting expression of miR-664 and miR-184 can reduce the cell migration capacity. Moreover, the flow cytometry of cells in each group after transfection revealed that the apoptosis rates in miR-664 and miR-184 inhibitor groups were increased, remarkably higher than those in blank and mimic groups. It can be preliminarily concluded through the in vitro experiment that inhibiting the expression of miR-664 and miR-184 can inhibit proliferation, migration and apoptosis of OS cells.

However, there were still some limitations in this study. First, the number of OS patients collected was small, so expression of miR-664 and miR-184 had no representative significance. Second, the downstream target genes of miR-664 and miR-184 were not deeply detected, and how they affected the downstream targets remains unknown. Therefore, it is hoped that in future research, the sample size can be increased, the downstream target genes can be detected and the mechanism can be explored, so as to complement the research content, thus further verifying results in this study and providing new methods and ideas for clinical diagnosis and treatment.

In conclusion, inhibiting the expression of miR-664 and miR-184 can promote apoptosis, inhibit the proliferation and reduce the migration of SOSP-9607 cells. miR-664 and miR-184 may provide a theoretical basis for the target selection in clinical targeted therapy and drug development for OS.

\section{Acknowledgements}

Not applicable.

\section{Funding}

No funding was received.

\section{Availability of data and materials}

The datasets used and/or analyzed during the present study are available from the corresponding author on reasonable request.

\section{Authors' contributions}

PT and JF were responsible for cell culture. QL helped with CCK-8 assay. WL and LY performed RT-qPCR. XZ extracted RNA. HN and PX contributed to performing statistical analysis. All authors read and approved the final manuscript.

\section{Ethics approval and consent to participate}

The study was approved by the Ethics Committee of Wuhan No. 1 Hospital (Wuhan, China). Patients who participated in this research had complete clinical data, and signed informed consents were obtained from the patients or guardians.

\section{Patient consent for publication}

Not applicable.

\section{Competing interests}

The authors declare that they have no competing interests.

\section{References}

1. Luetke A, Meyers PA, Lewis I and Juergens H: Osteosarcoma treatment - where do we stand? A state of the art review. Cancer Treat Rev 40: 523-532, 2014. 
2. Lagmay JP, Krailo MD, Dang H, Kim A, Hawkins DS, Beaty O 3rd, Widemann BC, Zwerdling T, Bomgaars L, Langevin AM, et al: Outcome of patients with recurrent osteosarcoma enrolled in seven phase II trials through Children's cancer group, Pediatric oncology group, and Children's oncology group: Learning from the past to move forward. J Clin Oncol 34 3031-3038, 2016.

3. Daw NC, Chou AJ, Jaffe N, Rao BN, Billups CA, Rodriguez-Galindo C, Meyers PA and Huh WW: Recurrent osteosarcoma with a single pulmonary metastasis: A multiinstitutional review. Br J Cancer 112: 278-282, 2015.

4. Subbiah V, Anderson P and Rohren E: Alpha emitter radium 223 in high-risk osteosarcoma: First clinical evidence of response and blood-brain barrier penetration. JAMA Oncol 1: 253-255, 2015.

5. Li J, Yang Z, Li Y, Xia J, Li D, Li H, Ren M, Liao Y, Yu S, Chen Y, et al: Cell apoptosis, autophagy and necroptosis in osteosarcoma treatment. Oncotarget 7: 44763-44778, 2016.

6. Bandara KV, Michael MZ and Gleadle JM: MicroRNA biogenesis in hypoxia. MicroRNA 6: 80-96, 2017.

7. Lee Y, Ahn C, Han J, Choi H, Kim J, Yim J, Lee J, Provost P, Rådmark O, Kim S, et al: The nuclear RNase III Drosha initiates microRNA processing. Nature 425: 415-419, 2003.

8. Park JK, Peng H, Yang W, Katsnelson J, Volpert O and Lavker RM: miR-184 exhibits angiostatic properties via regulation of Akt and VEGF signaling pathways. FASEB J 31: 256-265, 2017.

9. Wu L, Li Y, Li J and Ma D: MicroRNA-664 targets insulin receptor substrate 1 to suppress cell proliferation and invasion in breast cancer. Oncol Res, 2018 (Epub ahead of print). https://doi org $/ 10.3727 / 096504018 X 15193500663936$

10. Picci P: Osteosarcoma (osteogenic sarcoma). Orphanet J Rare Dis 2: 6, 2007.

11. Livak KJ and Schmittgen TD: Analysis of relative gene expression data using real time quantitative PCR and the 2(-Delta Delta C(T)) method. Methods 25: 402-408, 2001

12. Isakoff MS, Bielack SS, Meltzer P and Gorlick R: Osteosarcoma: Current treatment and a collaborative pathway to success. J Clin Oncol 33: 3029-3035, 2015.

13. Faisham WI, Mat Saad AZ, Alsaigh LN, Nor Azman MZ, Kamarul Imran M, Biswal BM, Bhavaraju VM, Salzihan MS, Hasnan J, Ezane AM, et al: Prognostic factors and survival rate of osteosarcoma: A single-institution study. Asia Pac J Clin Oncol 13: e104-e110, 2017

14. Chen YU, Xu SF, Xu M and Yu XC: Postoperative infection and survival in osteosarcoma patients: Reconsideration of immunotherapy for osteosarcoma. Mol Clin Oncol 3: 495-500, 2015.

15. Qi Y, Zhao C, Li H, Zhang B, Tada K, Abe H and Tada M: Genetic variations in interleukin- 6 polymorphism and the association with susceptibility and overall survival of osteosarcoma. Tumour Biol 37: 9807-9811, 2016.
16. Suzuki HI, Young RA and Sharp PA: Super-enhancer-mediated RNA processing revealed by integrative microRNA network analysis. Cell 168: 1000-1014.e15, 2017.

17. Chang TH, Tsai MF, Gow CH, Wu SG, Liu YN, Chang YL, Yu SL, Tsai HC, Lin SW, Chen YW, et al: Upregulation of microRNA-137 expression by Slug promotes tumor invasion and metastasis of non-small cell lung cancer cells through suppression of TFAP2C. Cancer Lett 402: 190-202, 2017.

18. Yu L, Wu D, Gao H, Balic JJ, Tsykin A, Han TS, Liu YD, Kennedy CL, Li JK, Mao JQ, et al: Clinical utility of a STAT3-regulated miRNA-200 family signature with prognostic potential in early gastric cancer. Clin Cancer Res 24: 1459-1472, 2018.

19. Li Y, Zhang M, Chen H, Dong Z, Ganapathy V, Thangaraju M and Huang S: Ratio of miR-196s to HOXC8 messenger RNA correlates with breast cancer cell migration and metastasis. Cancer Res 70: 7894-7904, 2010.

20. Yang H, Cho ME, Li TW, Peng H, Ko KS, Mato JM and Lu SC: MicroRNAs regulate methionine adenosyltransferase 1A expression in hepatocellular carcinoma. J Clin Invest 123: 285-298, 2013.

21. Park KS, Moon YW, Raffeld M, Lee DH, Wang Y and Giaccone G: High cripto-1 and low miR-205 expression levels as prognostic markers in early stage non-small cell lung cancer. Lung Cancer 116: 38-45, 2018.

22. Simonian M, Mosallayi M and Mirzaei H: Circulating miR-21 as novel biomarker in gastric cancer: Diagnostic and prognostic biomarker. J Cancer Res Ther 14: 475, 2018.

23. Bao Y, Chen B, Wu Q, Hu K, Xi X, Zhu W, Zhong X and Chen J: Overexpression of miR-664 is associated with enhanced osteosarcoma cell migration and invasion ability via targeting SOX7. Clin Exp Med 17: 51-58, 2017.

24. Ma C, Wei F, Xia H, Liu H, Dong X, Zhang Y, Luo Q, Liu Y and Li Y: MicroRNA-10b mediates TGF- $\beta 1$-regulated glioblastoma proliferation, migration and epithelial-mesenchymal transition. Int J Oncol 50: 1739-1748, 2017.

25. Lin BC, Huang D, Yu CQ, Mou Y, Liu YH, Zhang DW and Shi FJ: MicroRNA-184 modulates doxorubicin resistance in osteosarcoma cells by targeting BCL2L1. Med Sci Monit 22: 1761-1765, 2016

This work is licensed under a Creative Commons Attribution-NonCommercial-NoDerivatives 4.0 International (CC BY-NC-ND 4.0) License. 